\title{
Degrees That Are Not Degrees of Categoricity
}

\author{
Bernard Anderson and Barbara Csima
}

\begin{abstract}
A computable structure $\mathcal{A}$ is $\mathbf{x}$-computably categorical for some Turing degree $\mathbf{x}$ if for every computable structure $\mathscr{B} \cong \mathcal{A}$ there is an isomorphism $f: \mathscr{B} \rightarrow \mathcal{A}$ with $f \leq_{T} \mathbf{x}$. A degree $\mathbf{x}$ is a degree of categoricity if there is a computable structure $\mathcal{A}$ such that $\mathcal{A}$ is $\mathbf{x}$-computably categorical, and for all $\mathbf{y}$, if $\mathcal{A}$ is $\mathbf{y}$-computably categorical, then $\mathbf{x} \leq T \mathbf{y}$.

We construct a $\Sigma_{2}^{0}$ set whose degree is not a degree of categoricity. We also demonstrate a large class of degrees that are not degrees of categoricity by showing that every degree of a set which is 2-generic relative to some perfect tree is not a degree of categoricity. Finally, we prove that every noncomputable hyperimmune-free degree is not a degree of categoricity.
\end{abstract}

\section{Introduction}

Classically, isomorphic structures are considered to be equivalent. In computable structure theory, one has to be more careful. Different copies of the same structure may have different complexity, and for some structures, it can happen that there are two computable copies of the structure between which there is no computable isomorphism. In fact, for situations where this does not happen, we have the following definition.

Definition 1 A computable structure $\mathcal{A}$ is computably categorical if for all computable $\mathscr{B} \cong \mathcal{A}$ there exists a computable isomorphism between $\mathcal{A}$ and $\mathscr{B}$.

For example, any two computable dense linear orders without endpoints are computably isomorphic. Thus, any computable dense linear order without endpoints is computably categorical.

On the other hand, it is well known that the structure $(\mathbb{N},<)$, the natural numbers with the usual $<$ order, is not computably categorical. Indeed, let $\left\{K_{s}\right\}_{s \in \omega}$ be a

Received July 2, 2013; accepted February 6, 2014

First published online April 7, 2016

2010 Mathematics Subject Classification: Primary 03D30

Keywords: degree of categoricity, computably categorical, strong degree of categoricity, CatSpec, category spectrum, computable structure

(C) 2016 by University of Notre Dame DOI: 10.1215/00294527-3496154 


\title{
Degrees That Are Not Degrees of Categoricity
}

\author{
Bernard Anderson and Barbara Csima
}

\begin{abstract}
A computable structure $\mathcal{A}$ is $\mathbf{x}$-computably categorical for some Turing degree $\mathbf{x}$ if for every computable structure $\mathscr{B} \cong \mathcal{A}$ there is an isomorphism $f: \mathscr{B} \rightarrow \mathcal{A}$ with $f \leq_{T} \mathbf{x}$. A degree $\mathbf{x}$ is a degree of categoricity if there is a computable structure $\mathcal{A}$ such that $\mathcal{A}$ is $\mathbf{x}$-computably categorical, and for all $\mathbf{y}$, if $\mathcal{A}$ is $\mathbf{y}$-computably categorical, then $\mathbf{x} \leq T \mathbf{y}$.

We construct a $\Sigma_{2}^{0}$ set whose degree is not a degree of categoricity. We also demonstrate a large class of degrees that are not degrees of categoricity by showing that every degree of a set which is 2-generic relative to some perfect tree is not a degree of categoricity. Finally, we prove that every noncomputable hyperimmune-free degree is not a degree of categoricity.
\end{abstract}

\section{Introduction}

Classically, isomorphic structures are considered to be equivalent. In computable structure theory, one has to be more careful. Different copies of the same structure may have different complexity, and for some structures, it can happen that there are two computable copies of the structure between which there is no computable isomorphism. In fact, for situations where this does not happen, we have the following definition.

Definition 1 A computable structure $\mathcal{A}$ is computably categorical if for all computable $\mathscr{B} \cong \mathcal{A}$ there exists a computable isomorphism between $\mathcal{A}$ and $\mathscr{B}$.

For example, any two computable dense linear orders without endpoints are computably isomorphic. Thus, any computable dense linear order without endpoints is computably categorical.

On the other hand, it is well known that the structure $(\mathbb{N},<)$, the natural numbers with the usual $<$ order, is not computably categorical. Indeed, let $\left\{K_{s}\right\}_{s \in \omega}$ be a

Received July 2, 2013; accepted February 6, 2014

First published online April 7, 2016

2010 Mathematics Subject Classification: Primary 03D30

Keywords: degree of categoricity, computably categorical, strong degree of categoricity, CatSpec, category spectrum, computable structure

(C) 2016 by University of Notre Dame DOI: 10.1215/00294527-3496154 


\title{
Degrees That Are Not Degrees of Categoricity
}

\author{
Bernard Anderson and Barbara Csima
}

\begin{abstract}
A computable structure $\mathcal{A}$ is $\mathbf{x}$-computably categorical for some Turing degree $\mathbf{x}$ if for every computable structure $\mathscr{B} \cong \mathcal{A}$ there is an isomorphism $f: \mathscr{B} \rightarrow \mathcal{A}$ with $f \leq_{T} \mathbf{x}$. A degree $\mathbf{x}$ is a degree of categoricity if there is a computable structure $\mathcal{A}$ such that $\mathcal{A}$ is $\mathbf{x}$-computably categorical, and for all $\mathbf{y}$, if $\mathcal{A}$ is $\mathbf{y}$-computably categorical, then $\mathbf{x} \leq T \mathbf{y}$.

We construct a $\Sigma_{2}^{0}$ set whose degree is not a degree of categoricity. We also demonstrate a large class of degrees that are not degrees of categoricity by showing that every degree of a set which is 2-generic relative to some perfect tree is not a degree of categoricity. Finally, we prove that every noncomputable hyperimmune-free degree is not a degree of categoricity.
\end{abstract}

\section{Introduction}

Classically, isomorphic structures are considered to be equivalent. In computable structure theory, one has to be more careful. Different copies of the same structure may have different complexity, and for some structures, it can happen that there are two computable copies of the structure between which there is no computable isomorphism. In fact, for situations where this does not happen, we have the following definition.

Definition 1 A computable structure $\mathcal{A}$ is computably categorical if for all computable $\mathscr{B} \cong \mathcal{A}$ there exists a computable isomorphism between $\mathcal{A}$ and $\mathscr{B}$.

For example, any two computable dense linear orders without endpoints are computably isomorphic. Thus, any computable dense linear order without endpoints is computably categorical.

On the other hand, it is well known that the structure $(\mathbb{N},<)$, the natural numbers with the usual $<$ order, is not computably categorical. Indeed, let $\left\{K_{s}\right\}_{s \in \omega}$ be a

Received July 2, 2013; accepted February 6, 2014

First published online April 7, 2016

2010 Mathematics Subject Classification: Primary 03D30

Keywords: degree of categoricity, computably categorical, strong degree of categoricity, CatSpec, category spectrum, computable structure

(C) 2016 by University of Notre Dame DOI: 10.1215/00294527-3496154 


\title{
Degrees That Are Not Degrees of Categoricity
}

\author{
Bernard Anderson and Barbara Csima
}

\begin{abstract}
A computable structure $\mathcal{A}$ is $\mathbf{x}$-computably categorical for some Turing degree $\mathbf{x}$ if for every computable structure $\mathscr{B} \cong \mathcal{A}$ there is an isomorphism $f: \mathscr{B} \rightarrow \mathcal{A}$ with $f \leq_{T} \mathbf{x}$. A degree $\mathbf{x}$ is a degree of categoricity if there is a computable structure $\mathcal{A}$ such that $\mathcal{A}$ is $\mathbf{x}$-computably categorical, and for all $\mathbf{y}$, if $\mathcal{A}$ is $\mathbf{y}$-computably categorical, then $\mathbf{x} \leq T \mathbf{y}$.

We construct a $\Sigma_{2}^{0}$ set whose degree is not a degree of categoricity. We also demonstrate a large class of degrees that are not degrees of categoricity by showing that every degree of a set which is 2-generic relative to some perfect tree is not a degree of categoricity. Finally, we prove that every noncomputable hyperimmune-free degree is not a degree of categoricity.
\end{abstract}

\section{Introduction}

Classically, isomorphic structures are considered to be equivalent. In computable structure theory, one has to be more careful. Different copies of the same structure may have different complexity, and for some structures, it can happen that there are two computable copies of the structure between which there is no computable isomorphism. In fact, for situations where this does not happen, we have the following definition.

Definition 1 A computable structure $\mathcal{A}$ is computably categorical if for all computable $\mathscr{B} \cong \mathcal{A}$ there exists a computable isomorphism between $\mathcal{A}$ and $\mathscr{B}$.

For example, any two computable dense linear orders without endpoints are computably isomorphic. Thus, any computable dense linear order without endpoints is computably categorical.

On the other hand, it is well known that the structure $(\mathbb{N},<)$, the natural numbers with the usual $<$ order, is not computably categorical. Indeed, let $\left\{K_{s}\right\}_{s \in \omega}$ be a

Received July 2, 2013; accepted February 6, 2014

First published online April 7, 2016

2010 Mathematics Subject Classification: Primary 03D30

Keywords: degree of categoricity, computably categorical, strong degree of categoricity, CatSpec, category spectrum, computable structure

(C) 2016 by University of Notre Dame DOI: 10.1215/00294527-3496154 


\title{
Degrees That Are Not Degrees of Categoricity
}

\author{
Bernard Anderson and Barbara Csima
}

\begin{abstract}
A computable structure $\mathcal{A}$ is $\mathbf{x}$-computably categorical for some Turing degree $\mathbf{x}$ if for every computable structure $\mathscr{B} \cong \mathcal{A}$ there is an isomorphism $f: \mathscr{B} \rightarrow \mathcal{A}$ with $f \leq_{T} \mathbf{x}$. A degree $\mathbf{x}$ is a degree of categoricity if there is a computable structure $\mathcal{A}$ such that $\mathcal{A}$ is $\mathbf{x}$-computably categorical, and for all $\mathbf{y}$, if $\mathcal{A}$ is $\mathbf{y}$-computably categorical, then $\mathbf{x} \leq T \mathbf{y}$.

We construct a $\Sigma_{2}^{0}$ set whose degree is not a degree of categoricity. We also demonstrate a large class of degrees that are not degrees of categoricity by showing that every degree of a set which is 2-generic relative to some perfect tree is not a degree of categoricity. Finally, we prove that every noncomputable hyperimmune-free degree is not a degree of categoricity.
\end{abstract}

\section{Introduction}

Classically, isomorphic structures are considered to be equivalent. In computable structure theory, one has to be more careful. Different copies of the same structure may have different complexity, and for some structures, it can happen that there are two computable copies of the structure between which there is no computable isomorphism. In fact, for situations where this does not happen, we have the following definition.

Definition 1 A computable structure $\mathcal{A}$ is computably categorical if for all computable $\mathscr{B} \cong \mathcal{A}$ there exists a computable isomorphism between $\mathcal{A}$ and $\mathscr{B}$.

For example, any two computable dense linear orders without endpoints are computably isomorphic. Thus, any computable dense linear order without endpoints is computably categorical.

On the other hand, it is well known that the structure $(\mathbb{N},<)$, the natural numbers with the usual $<$ order, is not computably categorical. Indeed, let $\left\{K_{s}\right\}_{s \in \omega}$ be a

Received July 2, 2013; accepted February 6, 2014

First published online April 7, 2016

2010 Mathematics Subject Classification: Primary 03D30

Keywords: degree of categoricity, computably categorical, strong degree of categoricity, CatSpec, category spectrum, computable structure

(C) 2016 by University of Notre Dame DOI: 10.1215/00294527-3496154 


\title{
Degrees That Are Not Degrees of Categoricity
}

\author{
Bernard Anderson and Barbara Csima
}

\begin{abstract}
A computable structure $\mathcal{A}$ is $\mathbf{x}$-computably categorical for some Turing degree $\mathbf{x}$ if for every computable structure $\mathscr{B} \cong \mathcal{A}$ there is an isomorphism $f: \mathscr{B} \rightarrow \mathcal{A}$ with $f \leq_{T} \mathbf{x}$. A degree $\mathbf{x}$ is a degree of categoricity if there is a computable structure $\mathcal{A}$ such that $\mathcal{A}$ is $\mathbf{x}$-computably categorical, and for all $\mathbf{y}$, if $\mathcal{A}$ is $\mathbf{y}$-computably categorical, then $\mathbf{x} \leq T \mathbf{y}$.

We construct a $\Sigma_{2}^{0}$ set whose degree is not a degree of categoricity. We also demonstrate a large class of degrees that are not degrees of categoricity by showing that every degree of a set which is 2-generic relative to some perfect tree is not a degree of categoricity. Finally, we prove that every noncomputable hyperimmune-free degree is not a degree of categoricity.
\end{abstract}

\section{Introduction}

Classically, isomorphic structures are considered to be equivalent. In computable structure theory, one has to be more careful. Different copies of the same structure may have different complexity, and for some structures, it can happen that there are two computable copies of the structure between which there is no computable isomorphism. In fact, for situations where this does not happen, we have the following definition.

Definition 1 A computable structure $\mathcal{A}$ is computably categorical if for all computable $\mathscr{B} \cong \mathcal{A}$ there exists a computable isomorphism between $\mathcal{A}$ and $\mathscr{B}$.

For example, any two computable dense linear orders without endpoints are computably isomorphic. Thus, any computable dense linear order without endpoints is computably categorical.

On the other hand, it is well known that the structure $(\mathbb{N},<)$, the natural numbers with the usual $<$ order, is not computably categorical. Indeed, let $\left\{K_{s}\right\}_{s \in \omega}$ be a

Received July 2, 2013; accepted February 6, 2014

First published online April 7, 2016

2010 Mathematics Subject Classification: Primary 03D30

Keywords: degree of categoricity, computably categorical, strong degree of categoricity, CatSpec, category spectrum, computable structure

(C) 2016 by University of Notre Dame DOI: 10.1215/00294527-3496154 


\title{
Degrees That Are Not Degrees of Categoricity
}

\author{
Bernard Anderson and Barbara Csima
}

\begin{abstract}
A computable structure $\mathcal{A}$ is $\mathbf{x}$-computably categorical for some Turing degree $\mathbf{x}$ if for every computable structure $\mathscr{B} \cong \mathcal{A}$ there is an isomorphism $f: \mathscr{B} \rightarrow \mathcal{A}$ with $f \leq_{T} \mathbf{x}$. A degree $\mathbf{x}$ is a degree of categoricity if there is a computable structure $\mathcal{A}$ such that $\mathcal{A}$ is $\mathbf{x}$-computably categorical, and for all $\mathbf{y}$, if $\mathcal{A}$ is $\mathbf{y}$-computably categorical, then $\mathbf{x} \leq T \mathbf{y}$.

We construct a $\Sigma_{2}^{0}$ set whose degree is not a degree of categoricity. We also demonstrate a large class of degrees that are not degrees of categoricity by showing that every degree of a set which is 2-generic relative to some perfect tree is not a degree of categoricity. Finally, we prove that every noncomputable hyperimmune-free degree is not a degree of categoricity.
\end{abstract}

\section{Introduction}

Classically, isomorphic structures are considered to be equivalent. In computable structure theory, one has to be more careful. Different copies of the same structure may have different complexity, and for some structures, it can happen that there are two computable copies of the structure between which there is no computable isomorphism. In fact, for situations where this does not happen, we have the following definition.

Definition 1 A computable structure $\mathcal{A}$ is computably categorical if for all computable $\mathscr{B} \cong \mathcal{A}$ there exists a computable isomorphism between $\mathcal{A}$ and $\mathscr{B}$.

For example, any two computable dense linear orders without endpoints are computably isomorphic. Thus, any computable dense linear order without endpoints is computably categorical.

On the other hand, it is well known that the structure $(\mathbb{N},<)$, the natural numbers with the usual $<$ order, is not computably categorical. Indeed, let $\left\{K_{s}\right\}_{s \in \omega}$ be a

Received July 2, 2013; accepted February 6, 2014

First published online April 7, 2016

2010 Mathematics Subject Classification: Primary 03D30

Keywords: degree of categoricity, computably categorical, strong degree of categoricity, CatSpec, category spectrum, computable structure

(C) 2016 by University of Notre Dame DOI: 10.1215/00294527-3496154 


\title{
Degrees That Are Not Degrees of Categoricity
}

\author{
Bernard Anderson and Barbara Csima
}

\begin{abstract}
A computable structure $\mathcal{A}$ is $\mathbf{x}$-computably categorical for some Turing degree $\mathbf{x}$ if for every computable structure $\mathscr{B} \cong \mathcal{A}$ there is an isomorphism $f: \mathscr{B} \rightarrow \mathcal{A}$ with $f \leq_{T} \mathbf{x}$. A degree $\mathbf{x}$ is a degree of categoricity if there is a computable structure $\mathcal{A}$ such that $\mathcal{A}$ is $\mathbf{x}$-computably categorical, and for all $\mathbf{y}$, if $\mathcal{A}$ is $\mathbf{y}$-computably categorical, then $\mathbf{x} \leq T \mathbf{y}$.

We construct a $\Sigma_{2}^{0}$ set whose degree is not a degree of categoricity. We also demonstrate a large class of degrees that are not degrees of categoricity by showing that every degree of a set which is 2-generic relative to some perfect tree is not a degree of categoricity. Finally, we prove that every noncomputable hyperimmune-free degree is not a degree of categoricity.
\end{abstract}

\section{Introduction}

Classically, isomorphic structures are considered to be equivalent. In computable structure theory, one has to be more careful. Different copies of the same structure may have different complexity, and for some structures, it can happen that there are two computable copies of the structure between which there is no computable isomorphism. In fact, for situations where this does not happen, we have the following definition.

Definition 1 A computable structure $\mathcal{A}$ is computably categorical if for all computable $\mathscr{B} \cong \mathcal{A}$ there exists a computable isomorphism between $\mathcal{A}$ and $\mathscr{B}$.

For example, any two computable dense linear orders without endpoints are computably isomorphic. Thus, any computable dense linear order without endpoints is computably categorical.

On the other hand, it is well known that the structure $(\mathbb{N},<)$, the natural numbers with the usual $<$ order, is not computably categorical. Indeed, let $\left\{K_{s}\right\}_{s \in \omega}$ be a

Received July 2, 2013; accepted February 6, 2014

First published online April 7, 2016

2010 Mathematics Subject Classification: Primary 03D30

Keywords: degree of categoricity, computably categorical, strong degree of categoricity, CatSpec, category spectrum, computable structure

(C) 2016 by University of Notre Dame DOI: 10.1215/00294527-3496154 


\title{
Degrees That Are Not Degrees of Categoricity
}

\author{
Bernard Anderson and Barbara Csima
}

\begin{abstract}
A computable structure $\mathcal{A}$ is $\mathbf{x}$-computably categorical for some Turing degree $\mathbf{x}$ if for every computable structure $\mathscr{B} \cong \mathcal{A}$ there is an isomorphism $f: \mathscr{B} \rightarrow \mathcal{A}$ with $f \leq_{T} \mathbf{x}$. A degree $\mathbf{x}$ is a degree of categoricity if there is a computable structure $\mathcal{A}$ such that $\mathcal{A}$ is $\mathbf{x}$-computably categorical, and for all $\mathbf{y}$, if $\mathcal{A}$ is $\mathbf{y}$-computably categorical, then $\mathbf{x} \leq T \mathbf{y}$.

We construct a $\Sigma_{2}^{0}$ set whose degree is not a degree of categoricity. We also demonstrate a large class of degrees that are not degrees of categoricity by showing that every degree of a set which is 2-generic relative to some perfect tree is not a degree of categoricity. Finally, we prove that every noncomputable hyperimmune-free degree is not a degree of categoricity.
\end{abstract}

\section{Introduction}

Classically, isomorphic structures are considered to be equivalent. In computable structure theory, one has to be more careful. Different copies of the same structure may have different complexity, and for some structures, it can happen that there are two computable copies of the structure between which there is no computable isomorphism. In fact, for situations where this does not happen, we have the following definition.

Definition 1 A computable structure $\mathcal{A}$ is computably categorical if for all computable $\mathscr{B} \cong \mathcal{A}$ there exists a computable isomorphism between $\mathcal{A}$ and $\mathscr{B}$.

For example, any two computable dense linear orders without endpoints are computably isomorphic. Thus, any computable dense linear order without endpoints is computably categorical.

On the other hand, it is well known that the structure $(\mathbb{N},<)$, the natural numbers with the usual $<$ order, is not computably categorical. Indeed, let $\left\{K_{s}\right\}_{s \in \omega}$ be a

Received July 2, 2013; accepted February 6, 2014

First published online April 7, 2016

2010 Mathematics Subject Classification: Primary 03D30

Keywords: degree of categoricity, computably categorical, strong degree of categoricity, CatSpec, category spectrum, computable structure

(C) 2016 by University of Notre Dame DOI: 10.1215/00294527-3496154 


\title{
Degrees That Are Not Degrees of Categoricity
}

\author{
Bernard Anderson and Barbara Csima
}

\begin{abstract}
A computable structure $\mathcal{A}$ is $\mathbf{x}$-computably categorical for some Turing degree $\mathbf{x}$ if for every computable structure $\mathscr{B} \cong \mathcal{A}$ there is an isomorphism $f: \mathscr{B} \rightarrow \mathcal{A}$ with $f \leq_{T} \mathbf{x}$. A degree $\mathbf{x}$ is a degree of categoricity if there is a computable structure $\mathcal{A}$ such that $\mathcal{A}$ is $\mathbf{x}$-computably categorical, and for all $\mathbf{y}$, if $\mathcal{A}$ is $\mathbf{y}$-computably categorical, then $\mathbf{x} \leq T \mathbf{y}$.

We construct a $\Sigma_{2}^{0}$ set whose degree is not a degree of categoricity. We also demonstrate a large class of degrees that are not degrees of categoricity by showing that every degree of a set which is 2-generic relative to some perfect tree is not a degree of categoricity. Finally, we prove that every noncomputable hyperimmune-free degree is not a degree of categoricity.
\end{abstract}

\section{Introduction}

Classically, isomorphic structures are considered to be equivalent. In computable structure theory, one has to be more careful. Different copies of the same structure may have different complexity, and for some structures, it can happen that there are two computable copies of the structure between which there is no computable isomorphism. In fact, for situations where this does not happen, we have the following definition.

Definition 1 A computable structure $\mathcal{A}$ is computably categorical if for all computable $\mathscr{B} \cong \mathcal{A}$ there exists a computable isomorphism between $\mathcal{A}$ and $\mathscr{B}$.

For example, any two computable dense linear orders without endpoints are computably isomorphic. Thus, any computable dense linear order without endpoints is computably categorical.

On the other hand, it is well known that the structure $(\mathbb{N},<)$, the natural numbers with the usual $<$ order, is not computably categorical. Indeed, let $\left\{K_{s}\right\}_{s \in \omega}$ be a

Received July 2, 2013; accepted February 6, 2014

First published online April 7, 2016

2010 Mathematics Subject Classification: Primary 03D30

Keywords: degree of categoricity, computably categorical, strong degree of categoricity, CatSpec, category spectrum, computable structure

(C) 2016 by University of Notre Dame DOI: 10.1215/00294527-3496154 